DOI: 10.12957/demetra.2016.22229

\title{
Cultura material e alimentação: A evolução dos utensílios à mesa e a consolidação de comportamentos durante a refeição
}

\section{Material culture and food: The evolution of tableware and the consolidation of behaviours during the mealtime}

\author{
Jaqueline Sayuri Nishimural \\ Shirley Gomes Queiroz' \\ ' Universidade de Brasília, Instituto de Artes, \\ Departamento de Design. Brasília-DF, Brasil. \\ 0 conteúdo deste artigo é parte do projeto \\ desenvolvido pela autora na disciplina \\ "Diplomação em Projeto de Produto", do \\ Departamento de Design da Universidade de \\ Brasília, no segundo semestre de 2015. \\ Correspondência / Correspondence \\ Jaqueline Sayuri Nishimura \\ E-mail: j.sayuri.n@hotmail.com
}

\section{Resumo}

A alimentação não é a simples satisfação de uma necessidade nutricional e biológica, representa um meio de expressão cultural permeado por convenções que transmitem mensagens e em que imperam o bom comportamento. O compartilhamento de refeições também é fonte de prazer, e possui papel significativo na construção e solidificação de relações sociais. No âmbito da alimentação, é importante considerar não somente o tipo de alimento consumido, a quantidade ou a maneira como é preparado, mas deve-se atribuir igual relevância às circunstâncias do consumo. Os objetos utilizados, sua disposição à mesa, a forma de manipulação, ordem de uso, entre outros protocolos, refletem simbologias herdadas ao longo dos séculos e moldam o contato do comensal com o alimento. O presente artigo propõe que o interagir contextual com os utensílios empregados na alimentação consolida progressivamente certos hábitos e colabora na construção identitária, na medida em que variações na cultura material resultam em formas distintas de se lidar com o alimento. Utiliza-se uma aproximação do ponto de partida europeu, por sua vasta influência no Brasil e no mundo em decorrência dos modos e produtos difundidos no período de expansão marítima e colonização. Além de uma análise da evolução e diversificação dos aparatos utilizados nas refeições, e o intrínseco desenvolvimento das regras de etiqueta, segue-se uma breve exposição das influências de tais costumes na mesa do brasileiro.

Palavras-chave: Alimentação. Etiqueta. Cultura material. Hábito. Refeição. 


\section{Abstract}

Consuming food is not the simple satisfaction of a nutritional and biological need, but it is also a mean of cultural expression, as it is permeated by conventions that stipulate behaviour standards and convey messages. The sharing of a meal is also a source of pleasure, and it plays a significant role in building and in the consolidation of social relations. The context of food eating, it is important to consider that, not only the type of food consumed, the amount or the way it is prepared, but also the circumstances of consumption should be given an equal importance. The utensils, their disposal at the table, the way of handling, order of use, among other protocols reflect symbologies inherited over the centuries and contribute to shape the contact between diner and food. This article proposes that the contextual interaction with the utensils applied in food consumption gradually consolidates certain habits and collaborates in identity construction, as variations in material culture result in distinct ways of dealing with food. An approach from a European starting point is taken, for its vast influence both in Brazil and worldwide, since the period of maritime expansion and colonisation, in which their habits and products were widespread. In addition to an analysis of the evolution and diversification of the apparatuses used during the meals, and the intrinsic development of etiquette rules, it follows a brief exposition of the influences of such manners on the Brazilian table.

Key words: Alimentation. Etiquette. Material culture. Habit. Meal.

\section{Introdução}

A alimentação, a respiração, o descanso e a reprodução constituem os elementos básicos para a sobrevivência e perpetuação do homem na Terra. Entretanto, a alimentação não pode ser encarada apenas como um ato nutricional, ela também é veículo de expressão cultural na maneira que convém e impõe o controle de impulsos.

O homem transforma o consumo do alimento, que é uma necessidade biológica, em uma necessidade cultural, usando o ato de comer como um condutor para relacionamentos sociais. ${ }^{1}$ Isso justifica a presença de uma série de mecanismos de integração e distinção social relativos às regras que permeiam uma refeição, criando a premência de domínio do que se consideram boas maneiras à mesa para que seja garantida a inclusão no meio. Nesse sentido, Carneiro ${ }^{2}$ postula que "o que se come é tão importante quanto quando se come, onde se come, como se come e com quem se come". 
Segundo Fourier apud Carneiro, ${ }^{3}$ comer também é um ato cognitivo, visto que envolve atenção, percepção, memória, raciocínio, imaginação, pensamento e juízo. A alimentação é interculturalmente definida por variações de mentalidades, ritos, proibições dietéticas e religiosas, valores éticos, transmissão de comportamentos inter e intrageração, regras de etiqueta, gestos, psicologias individuais e coletivas, e muitos outros fatores. ${ }^{4}$

Os ingredientes usados no preparo de alimentos - desde o rompimento do isolamento continental pelas rotas terrestres e marítimas de comércio - não mais diferenciam culturas, mas a maneira como se prepara e se come, ainda nos dias de hoje, sim. Portanto, entende-se que os padrões de permanências e mudanças dos hábitos e práticas alimentares encontram suas referências na própria dinâmica social: constantemente recebendo, adaptando e hibridizando influências.

Os significados dos rituais de alimentação também encontram forte meio de expressão na cultura material. A própria maneira de se utilizar talheres para encaminhar o alimento à boca sinaliza a convenção de um comportamento e não de outro, como o de levar o alimento à boca com as mãos, com o auxílio de outro tipo de utensílio, ou sorvendo-o diretamente do recipiente. $\mathrm{Eco}^{5}$ aborda o tema da seguinte maneira:

Usar uma colher para levar um alimento à boca ainda é a execução de uma função através do emprego de um artefato que a permite e a promove: mas dizer que o artefato 'promove' a função indica que também ele assume uma função comunicacional, comunica a função a executar; ao mesmo tempo o fato de alguém usar a colher, aos olhos da sociedade que o observa, já se torna a comunicação a uma adequação sua a certos usos.

Desse modo, é importante ressaltar que a maneira de se portar à mesa - como são manuseados os utensílios, a postura, os gestos, entre outros - não é comportamento adotado automaticamente, mas resultado de construções sociais elaboradas para enviar mensagens. Regras de etiqueta são adotadas em torno de ocasiões e objetos com o intuído de normatizar posturas a fim de tornar o convívio agradável e cível. Nesse âmbito, os protocolos apropriam-se de linguagens não verbais - gestuais, simbólicas e códigos - que moldam a interação dos convivas à mesa entre si e, principalmente, com o alimento servido.

\section{A evolução e complexificação dos utensílios nas refeições}

A palavra 'talher' vem de tailhoir, do francês, e significa 'prato de cortar carne'. ${ }^{6} \mathrm{O}$ termo passou então a designar variações de utensílios utilizados à mesa para cortar e servir alimentos. As formas mais populares: o garfo, a faca e a colher, tiveram caminhos muito distintos ao longo da história, e só foram popularizados da maneira como são utilizados hoje durante o século VXIII. 
Compreender o contexto histórico e cultural desses utensílios é de vital importância para verificar a permanência de certos hábitos à mesa.

Especula-se que a maior parte dos alimentos era consumida com as mãos ou com colheres até o final do século XV. No século XVI, a colher assumiu grande importância em toda a Europa, pois grande parte da comida era servida em pequenos pedaços ou sob a forma de ensopados. ${ }^{7}$ Ainda de acordo com o autor, no período da Idade Média, em geral, os anfitriões de banquetes proviam somente algumas colheres, que eram compartilhadas entre todos os comensais.

A faca, como é conhecida hoje, provavelmente surgiu na Idade do Bronze, a partir de 3300 a.C. ${ }^{8}$ Durante muito tempo, não houve distinção entre facas utilizadas para a caça ou para trinchar e as que se usavam à mesa. Estas eram pontiagudas e serviam também para espetar os alimentos nas bandejas compartilhadas. Franco ${ }^{7}$ afirma que, já na Idade Média, apenas os nobres possuíam facas especiais para cortar sua comida e eram consideradas objetos de uso pessoal, sendo transportadas em kits de viagem, ocasionalmente acompanhadas de colheres.

De acordo com Jones, ${ }^{9}$ a presença de facas à mesa era uma ameaça constante. Especialmente porque significantes fontes de hidratação durante as refeições eram o vinho e a cerveja, o que favorecia acidentes e comportamentos violentos. Sobre o uso da faca à mesa, Norbert Elias, ${ }^{10}$ em sua obra O Processo Civilizador, escreve: "A faca, pela própria natureza de seu uso social, reflete mudanças na personalidade humana, com suas mutáveis compulsões e desejos. Ela é a materialização de situações históricas e de fidelidades estruturais da sociedade."

A ponta afiada das facas perdeu o sentido com a crescente popularização do garfo ao longo do século XVII. Em 1669, o rei Louis XIV da França bane as facas pontudas da mesa, favorecendo o aperfeiçoamento do utensílio para comer. ${ }^{11}$ Segundo Visser, ${ }^{1}$ a maneira de segurar a faca evoluiu com a sua especialização para a mesa: esta passa a ter apenas um lado cortante, servindo o lado cego de apoio para o dedo indicador, considerado o modo mais refinado de segurar o instrumento.

É importante considerar que o uso individual do conjunto faca e garfo coincide com a evolução de pratos mais planos, visto que requerem uma superfície lisa e dura para serem manipulados confortavelmente. ${ }^{12}$ Os pratos individuais começaram a surgir durante o século XVII, feitos sobretudo de porcelana, madeira e de metais como o estanho e a prata. ${ }^{13}$ Pratos rasos de cerâmica já eram razoavelmente comuns na França, ao final do século XVII, mas só foram consolidados como substituídos das tigelas à mesa no século XIX. ${ }^{1}$

Os usos mais antigos atribuídos ao garfo remetem ao antigo Egito e à cultura Qijia, que residiu até 1900 a.C., onde hoje é parte da China. O livro bíblico de I Samuel 2:13, escrito por volta de 640-540 a.C., postula que os assistentes dos sacerdotes judeus usavam garfos em rituais de sacrifício de animais. ${ }^{14}$ Mas o costume dos europeus ocidentais de utilizar garfos à mesa foi relativamente 
tardio, começando na Itália somente no início do século XI e sendo popularizado em outras áreas da Europa apenas no século XVII.

O primeiro garfo registrado na Europa Central data de 1004 d.C., quando a princesa de origem bizantina Maria Argyropoulina casa-se com um nobre italiano, trazendo consigo um estojo com garfos de ouro. ${ }^{11}$ Seu refinamento, visto como excessivo, foi tratado como herético, como apontam os relatos de Wright apud Snodgrass: ${ }^{15}$

[...]tamanho era o luxo de seus hábitos [...] que ela se negava a tocar a comida com seus dedos, mas comandaria seus eunucos para cortá-la em pequenos pedaços, os quais seriam empalados em um certo instrumento de ouro com dois dentes, e então carregados à sua boca.

A morte da princesa, dois anos depois, por uma praga epidêmica, foi encarada como castigo divino. Especula-se que o problema com o uso do garfo se deve à sua semelhança com o iconográfico forcado do demônio. ${ }^{15}$ Com o tempo, o mesmo passou a ser aceitável em usos ocasionais, restrito para alimentos com molho, grudentos ou pegajosos, como as sobremesas, e principalmente para as mulheres.

Introduzido na França, em 1540, por Catarina de Médici, o garfo se tornou um sucesso por diferenciar ricos de pobres, que comiam com a mão. Nessa época, o garfo era um artigo de luxo, e somente nobres possuíam um jogo de talheres, que viajavam com eles em estojos que podiam ser pendurados sob o ombro ou em volta da cintura. ${ }^{14}$

A lentidão da incorporação dos garfos à mesa é também justificada pelo fato de eles não funcionarem bem. Inicialmente, possuíam apenas dois dentes, que não eram suficientes para segurar a comida. ${ }^{11}$ No início do século VXIII, a revolução dos napolitanos acrescentou o quarto dente ao garfo para facilitar o ato de enrolar os fios de espaguete no talher, dando a forma que conhecemos hoje. ${ }^{8}$

O garfo passou a ficar mais popular à medida que os ideais de higiene mudaram, pois começaram a ser vistos benefícios como manter tanto as mãos como o alimento limpos, e livre de contato com os outros. Talvez tenha sido um reflexo de proteção contra as pragas, ${ }^{16}$ nessa época ainda era comum, por exemplo, que os comensais escarrassem na mão ou na toalha de mesa, usando-a também para limpar os talheres compartilhados.

Na Europa, o uso de talheres também está frequentemente associado a mudanças significantes nas tendências de moda - como, por exemplo, a introdução de golas altas, babados exuberantes, mangas amplas e longas, além dos french cuffs -, o que tornou comer com as mãos altamente desconfortável e inconveniente. 
Apenas ao longo do século XVII as classes dominantes se iniciaram na pratica de comer com seus próprios talheres em pratos individuais, e o garfo passa a ser utilizado em conjunto com a faca, que continua sendo o utensílio principal, utilizado na mão direita. O individualismo começa então a ser demarcado pelo conjunto de artefatos organizados a uma distancia absolutamente regular dos vizinhos de mesa. ${ }^{13}$

No final do século XVII surgem faqueiros com colheres, facas e garfos. A produção de talheres em larga escala na Inglaterra, depois de 1650, teve papel importante na melhoria das maneiras à mesa. ${ }^{17}$ Porém, somente no início do século XVIII é que se começam a adquirir múltiplos conjuntos de talheres, e segundo Goldsmith, ${ }^{14}$ a prataria de mesa passa a ser popular na Europa, para aqueles que podiam pagar.

De acordo com Lima, ${ }^{13}$ no século XIX, o movimento de maior individualização e especialização de objetos atinge o domínio da alimentação em todas as suas expressões. Progressivamente, o aparelhamento de jantar se completa com sopeiras, molheiras, fruteiras, cremeiras, entre outros. Solidificam-se os três formatos diferentes de pratos: fundos, rasos e sobremesa, adequando-se ao modelo prevalente da refeição em três cursos. Os talheres também especializam-se em formas menores destinadas à sobremesa, chá e café, modelos especiais para queijos, frutas, ostras e peixes. Até mesmo copos e taças têm seus formatos ajustados aos diferentes tipos de bebidas servidos durante a refeição.

Finalmente, no século XX, a invenção do aço inox possibilita a criação de talheres fáceis de produzir e manter, favorecendo a popularização dos mesmos nas colônias e assentamentos fora da Europa.

\section{O desenvolvimento dos protocolos à mesa}

Dado o contexto histórico da incorporação dos talheres e outros artefatos à mesa, é importante destacar que estes "não foram inventados como utensílios técnicos com finalidades óbvias e instruções claras de uso. No decorrer de séculos, na relação social e no emprego direto, suas funções foram gradualmente sendo definidas e suas formas investigadas e consolidadas". ${ }^{10}$ Todos os movimentos da mão - por exemplo, a maneira como segurar e movimentar os talheres - foram padronizados apenas gradualmente, ${ }^{10}$ sendo criadas outras convenções relativas ao seu uso. Desse modo, entende-se que os comportamentos à mesa codificaram-se paralelamente ao processo de disseminação dos aparatos auxiliares da refeição. 
Figueiredo expõe uma definição interessante do conceito de etiqueta, que pode ser acatada no âmbito dos protocolos à mesa (apud Nakagawa ${ }^{18}$ ):

A etiqueta trata de regras que regem o comportamento do ser social. É uma maneira de se conduzir de acordo com normas predeterminadas em uma sociedade visando ser agradável aos outros. Tais regras são transmitidas por meio de gestos, modos de falar, atitudes, apresentação, visual adequado e seu significado mais profundo demonstrado pelo grau de cortesia e humanidade.

A noção de 'habitus', de Pierre Bourdieu, também contribui para o entendimento de tais normas: é um conjunto de 'disposições' que não são autodeterminadas ou predeterminadas por fatores ambientais externos. Elas tendem a operar em um nível subconsciente e são "incutidas através de experiências e ensinamentos explícitos", sendo produzidas e reproduzidas por interações sociais. ${ }^{19}$

Admite-se que muitos hábitos cultivados à mesa, atualmente, tiveram sua origem por volta do século V através de obras da religião judaica - o Talmude.${ }^{20} \mathrm{Na}$ época da Renascença, a Itália liderou a Europa no que diz respeito a cortesias e boas maneiras. Apesar de ser priorizada a difusão de boas maneiras no âmbito familiar, os manuais de civilidade ganharam maior difusão em 1440, com a invenção da imprensa pelo alemão Johannes Gutenberg. ${ }^{20}$ No período entre os séculos XIII e XVI, obras literárias de grande destaque já enfatizavam o que seriam os comportamentos mais adequados durante as refeições, que possuíam papel de destaque nas relações sociais. Manuais de alta relevância para o ensinamento de boas maneiras à mesa foram:

- As cinquenta cortesias para a mesa, de 1290, escrito por Fra Bonvincio da Riva.

- O livro do cortesão (também conhecido como The Courtier ou O cortesão), de 1507, escrito por Baldassare Castiglione.

- Galateo, de 1558, escrito por Giovanni della Casa.

Segundo Lima, ${ }^{13}$ "no século XVI, ao se esboçar com as monarquias absolutas uma nova aristocracia e uma hierarquia social mais rígida, foi aumentando o rigor em relação às condutas, que se tornaram pouco a pouco mais refinadas à mesa”. Isso implicou o desenvolvimento de protocolos relacionados à ordem e lugares para se sentar, aos utensílios e suas respectivas etiquetas, à linguagem do corpo e à conversação.

No entanto, a mudança prática mais significativa foi a individualização do ato de comer. A forma de se sentar à mesa, a introdução do garfo e do prato de uso pessoal revelaram uma mudança de perspectiva não só em relação à comida, mas também o crescimento de um movimento social de distanciamento e individualização. ${ }^{16}$ 
As regras de etiqueta também foram revolucionadas pela refeição em cursos introduzidos pelos franceses. Os pratos variados seriam consumidos segundo a natureza dos alimentos e combinados com a bebida apropriada, em consonância com os estágios fisiológicos sucessivos que se desenvolvem no organismo ao longo da refeição. ${ }^{21} \mathrm{~A}$ influência da cultura alimentar francesa estendeu-se com vigor nos quesitos estéticos - na apresentação dos pratos e na louçaria -, que ritualizavam os comportamentos no momento da refeição, sendo esta "um complexo artisticamente arquitetado para servir como meio de pertencimento identitário a determinado grupo social", ${ }^{22}$ tornando-se a principal referência na Europa e em outros países do mundo no século XVIII.

Considerando as mudanças na cultura material nos séculos XVIII e XIX, Lima ${ }^{13}$ defende que a especialização de louças e talheres somou-se à "progressiva complexificação do ritual do jantar, ao protocolo paulatinamente mais rígido, à cada vez mais intrincada codificação dos gestos e dos movimentos do corpo, atestando a reordenação de todo o subsistema alimentar”. Em um dado momento, já não bastava fornecer e utilizar faca, garfo e colher no lugar das mãos, mas passou a ser requisitada uma troca de louças e talheres especializados entre as etapas da refeição. Outro reflexo das mudanças nos rituais de alimentação na Europa é a criação da sala especial para refeições; posterior aos meados do século XVIII. Antes estas eram servidas em salas com outras funções. ${ }^{7}$

A nova compartimentação da unidade doméstica e o surgimento da sala de jantar; a especialização do seu mobiliário e adequação às funções de exibir e guardar alfaias, oferecer e ingerir alimentos; sua disposição e ordenação espacial; as alterações no modo de servir e na estruturada refeição; as formas de comportamento à mesa e a sofisticação do seu equipamento, todas essas mudanças na cultura material foram parte de um mesmo processo, tendo sido socialmente produzidas para transmitir mensagens. ${ }^{13}$

Ainda nos dias de hoje, a disposição dos objetos à mesa reflete simbologias herdadas ao longo dos séculos. $\mathrm{O}$ ato de virar o fio de corte da faca para dentro vem da Idade Média. A intenção do anfitrião é mostrar que está desarmado, emitindo um sinal de confiança e paz para os convidados. ${ }^{23}$ Outro resquício dos tempos de violência à mesa é a posição de descanso dos talheres. Não gesticular com os mesmos na mão e abaixar a faca quando esta não está em uso era associado a altas maneiras. ${ }^{24}$

O fato de o garfo ficar na mão esquerda e a faca na mão direita vem dos tempos do rei Luís XIV. A ordem persiste até hoje, porque todas as regras de etiqueta foram concebidas para pessoas destras, visto que os canhotos eram discriminados. ${ }^{23}$ Os talheres são sempre descansados e deixados no prato - e não sob a mesa -, porque a introdução de garfos e guardanapos individuais elevou os padrões de higiene e limpeza no momento da refeição, que passou a não admitir máculas fora da área destinada aos alimentos, nem mesmo na toalha de mesa. 


\section{A abrangência do uso de aparatos ao modo europeu e a percepção de 'etiqueta'}

Os utensílios de mesa não podem ser entendidos apenas em termos de função - uma vez que há muito pouco que se possa fazer com eles que não se possa fazer diretamente com as mãos -, mas devem ser compreendidos em seus significados socialmente construídos. ${ }^{12}$ A maneira como esses artefatos são manuseados sempre esteve atrelada a uma série de mecanismos de diferenciação entre culturas ou entre classes dentro de uma mesma cultura.

Os códigos estabelecidos pelos 'manuais de civilidade' entre os séculos XVI e XVIII tinham como finalidade a segregação social e a garantia de compartilhar momentos com aqueles que estivessem imbuídos de instruções semelhantes em todos os setores da vida. ${ }^{25}$

Gradativamente, os talheres foram se fortalecendo como mediadores entre o 'estado de natureza' e 'o estado de cultura'. ${ }^{13} \mathrm{O}$ ato de usar exclusivamente as mãos para levar o alimento à boca passou a ser associado ao desmazelo e à falta de maneiras. Nessa perspectiva, Lima ${ }^{13}$ ressalta que no século XVIII as recomendações dos manuais de civilidade ainda enfatizavam a não utilização das mãos - frisando que apenas os canibais comiam com os dedos.

De acordo com Carneiro, ${ }^{2}$ em um âmbito mais sutil, os hábitos e costumes ligados à alimentação também contribuíram para a revolução silenciosa que constitui o 'processo civilizatório', no qual a etiqueta à mesa ocupou papel de destaque ao introduzir novos costumes, como a adoção do guardanapo, do prato como a base sob a qual é colocada o alimento e o uso dos talheres. $\mathrm{O}$ processo civilizatório - que nada mais é do que a difusão da cultura e valores europeus - deu-se majoritariamente pelos regimes de colonização e comércio, ambos impulsionados pela Revolução Industrial. Em parte, também foi difundido pela emigração de europeus, estimulada por conflitos religiosos, étnicos, territoriais e pela falta de recursos e alimentos.

Os produtos e as maneiras ocidentais à mesa se espalham por meio de comunidades europeias que se estabelecem em diversos pontos da África, Ásia, América e Oceania, principalmente ao longo do século XIX, que marcou o apogeu dos padrões burgueses. Essa difusão acabou por ser de mão dupla, tornando-se uma fusão com os produtos e costumes locais. ${ }^{3}$

Em muitos países asiáticos, os talheres europeus foram adotados pelo Estado como símbolos de modernidade. No século XX, sob influência russa, o uso de garfos e colheres se tornou comum nos países soviéticos da Ásia Central. Nota-se que, nesses casos, a proliferação desses utensílios partiu de ambientes urbanos e de fontes centrais de poder. ${ }^{26}$ No que diz respeito aos fatores simbólicos que acompanham o uso das louças e talheres, a ampla popularização desses itens contribuiu para a diluição de alguns significados e rituais, favorecendo a adaptação e a criação de outros. 
Atualmente, a maneira como as regras de etiqueta são percebidas sofreu forte influência da chamada indústria cultural. Sua assimilação pela mídia, voltada ao público em massa, resultou em um enfraquecimento da compreensão da etiqueta como prática associada exclusivamente às camadas superiores da população. ${ }^{25}$

O domínio das regras de etiqueta encontra-se hoje associado à inteligência social de um indivíduo, pois revela respeito e consideração para com os demais. A partir daí, desenvolveu-se o ideal de etiqueta 'útil' tanto na vida pessoal, quanto profissional, que enfatiza a necessidade de um refinamento enfocado como marketing pessoal. Nesse sentido, o domínio de boas maneiras, principalmente à mesa (visto que esse é um ambiente onde se criam e solidificam relações), pode ser um diferencial, conferindo certo destaque ao praticante.

Nakagawa ${ }^{18}$ afirma que esse mecanismo cultural nutre o imaginário social sobre o que constitui as boas e elevadas maneiras e fomenta o consumo da etiqueta como bem simbólico, favorecendo o desenvolvimento de livros, websites, programas de televisão, vídeos e a procura de profissionais especializados em tais protocolos.

\section{A incorporação dos modos europeus à mesa no Brasil}

Até onde essas influências se estenderam - e ainda se estendem - no Brasil? O evento definidor da intensificação dos modos europeus no Brasil foi a chegada de membros da Família Real, em 1808. Temendo a invasão de Napoleão, o Príncipe D. João foi transferido para o Brasil com uma caravana de aproximadamente quinze mil pessoas. ${ }^{8}$ Junto com esta caravana de nobres também vieram os diversos ingredientes, hábitos e utensílios sofisticados para a alimentação, a fim de servir à corte como agradasse. Braga ${ }^{27}$ afirma que:

A retirada da Corte para o Rio de Janeiro e a referida abertura do Brasil ao exterior favoreceram a circulação e a adaptação de novas modas e hábitos à mesa e estimularam a adoção de novos consumos, especialmente visíveis na capital.

No início do século XIX, os hábitos franceses ganhavam espaço na rotina e nos eventos sociais da corte e da aristocracia. Além do Rio de Janeiro, outras cidades brasileiras também foram influenciadas pela gastronomia francesa e seu arcabouço de comportamentos à mesa. ${ }^{22} \mathrm{Tal}$ movimento, contudo, não se deu de maneira uniforme em todas as regiões.

Nesse período, a etiqueta à mesa variava com os grupos sociais. As populações do litoral estavam mais próximas dos hábitos europeus devido ao contato mais frequente com estrangeiros e artefatos importados. Porém, o mesmo não se passava no interior, onde o os mais comuns utensílios de mesa ainda eram desconhecidos. ${ }^{27}$ 
No Brasil do século XIX, tal como na Europa, faziam-se refeições em casa, nas estalagens, hotéis, restaurantes, cafés, casas de pasto, botequins, armazéns, lojas de bebidas, tabernas e pela rua. ${ }^{27}$ Diante disso, Lima ${ }^{13}$ propõe que dois modelos de alimentação foram simultaneamente adotados, em domínios opostos:

[...] emergiram dois perfis claramente diferenciados de comportamento: um, resultante da importação do modelo franco-inglês, adotado pelas camadas mais altas da sociedade para 'consumo externo', visando seu próprio reconhecimento e legitimação. O outro, inequivocadamente relacionado ao processo colonizador e fortemente influenciado pelos hábitos portugueses, acabou constituindo o modelo por excelência para 'consumo interno', compondo o dia-a-dia dos segmentos médios da população.

Ao final do século XIX, as estruturas de jantar franco-inglesas passam a ser seguidas com mais afinco em Portugal, que assim como o Brasil, foi marcado pelo hibridismo no que se refere à alimentação. De maneira análoga, a alimentação no Brasil assume ares culturais nesse período. "O espaço privado vai sendo privilegiado concomitantemente à expansão da cultura burguesa. $\mathrm{O}$ centro da sociabilidade se transfere para a sala de jantar, marcando um espaço de transição entre o público e o privado", relata Strong (apud Soares e Corção ${ }^{22}$ ).

A febre de consumo de utensílios de mesa nos séculos XVIII e XIX intensificou a assimilação dos hábitos europeus à mesa. O Brasil recebeu o impacto brutal da expansão das manufaturas inglesas, após a abertura dos portos.

O pais foi inundado pelas exuberantes faianças decalcadas e em sua ânsia de identificação com os valores franco-ingleses, em busca de reconhecimento, a sociedade foi absorvendo gradativamente as formas de comportamento delas indissociadas. Mesclando-as de modo peculiar às suas próprias tradições, fortemente calcadas nos hábitos dos colonizadores, criou expressões hibridas, marcadas por acentuados contrastes. ${ }^{13}$

Diante desse cenário, é evidente a variedade de estilos e influências que se alternaram no Brasil durante e mesmo após a colonização, que certamente contribuíram para a formação de uma identidade híbrida à mesa.

Atualmente, observa-se que a alimentação mantém um papel central nas relações sociais e nos momentos de lazer do brasileiro. Da Matta (apud Santos ${ }^{4}$ ), defende que "a comida tem o papel de destacar identidades e, conforme o contexto das refeições, elas podem ser nacionais, regionais, locais, familiares ou pessoais". Quanto aos protocolos primordialmente europeus impostos ou assimilados durante o período de colonização, Matarazzo ${ }^{28}$ afirma que "num país jovem, sem tradições milenares, como o Brasil, e em dias como os de hoje, em que a comunicação transforma rapidamente os conceitos, não se espera um rigor britânico no que diz respeito à etiqueta e ao comportamento". 


\section{Considerações finais}

A conjuntura de tantos rituais e significados relacionados à alimentação justifica-se pela sua necessidade inerente a subsistência humana. Observa-se que, desde os primórdios de sua implementação, os utensílios relacionados à alimentação formalizam certas maneiras de comer. Jeudy $^{29}$ defende que a coesão de um objeto advém da associação em série de 'representações estáveis' que marcam sua história. Dessa forma, entende-se que os objetos apropriaram-se dos significados hoje conhecidos somente pelo interagir contextual, ${ }^{30}$ ou seja, as qualidades de um artefato não são intrínsecas, mas atribuídas de acordo com um repertório cultural e experiências, que estabelecem que um dado significante denota um determinado significado. ${ }^{5}$ Esse processo é pautado em comportamentos sociais, que priorizam uns em detrimento de outros.

Desde sua estabilização no século XIX, o manuseio de louças, talheres, guardanapos e outros utensílios à mesa permaneceu quase imutável em seus aspectos essenciais, ou funções primeiras. De acordo com Norbert Elias, "até mesmo o surgimento da tecnologia em todas as áreas - inclusive na cozinha - deixou virtualmente inalteradas as técnicas à mesa e outras formas de comportamento”.

Contudo, ressalta-se que tanto os aparatos como os modos contemporâneos à mesa são produtos da miscigenação cultural, revelando vestígios de trocas e adaptações. Ainda que se tenha adotado uma abordagem de centro europeu, é importante considerar que a Europa também recebeu inúmeras influências de outros povos até atingir um arcabouço de utensílios e hábitos "estáveis", passados adiante pelo comércio e colonização - que prosseguiu em uma via de mão dupla em termos de influências.

Mais adiante, Santos ${ }^{4}$ afirma que "a pós-modernização embalada pela globalização tem imposto novas formas de consumo alimentar, novos costumes, hábitos e padrões alimentares", alterando, assim, convenções historicamente pautadas pela tradição e pelos costumes. Então, infere-se que, apesar de aparentemente estáveis, a permanência de certos rituais, comportamentos, aparatos, e até mesmo o ambiente - entre outros fatores ligados ao momento das refeições -, estão constantemente sujeitos a mudanças. As alterações no sistema da alimentação podem ser ocasionadas pela assimilação ou hibridização de costumes externos ou pelo próprio reflexo de mudanças sociais internas - como, por exemplo, a alteração na gestão do tempo destinado à alimentação.

Portanto, cultura material e comportamento à mesa são aspectos indissociáveis da prática alimentar. Os objetos definem não somente a apresentação e forma de consumo dos mantimentos, mas também percepções em relação à comida servida, definindo interação, formalidade, sacralidade, e ajudando a formar juízos de valor figurativo, como o julgamento de civilidade e demais associações culturais. 


\section{Referências}

1. Visser M. The rituals of dinner: the origins. Evolution, eccentricities and meaning of table manners. New York: Grove Weidenfeld; 1991.

2. Carneiro HS. Comida e sociedade: uma história da alimentação. Rio de Janeiro: Campus; 2003.

3. Carneiro HS. Comida e sociedade: significados sociais na história da alimentação. História Questões \& Debates 2005; 42(1):71-80.

4. Santos CRA. A alimentação e seu lugar na história: os tempos da memória gustativa. História Questões \& Debates 2005; 42:11-31.

5. Eco U. A estrutura ausente. São Paulo: Perspectiva; 1997. 190 p.

6. Cobra RQ. Mesa de refeições: seu aparelhamento e o uso dos talheres [Internet]. Brasília: Cobra Pages; 2001. Disponível em: http://www.cobra.pages.nom.br/bmp-mesatalheres.html

7. Franco A. De caçador a gourmet: uma história da gastronomia. Brasília: Thesaurus; 2001. 238 p.

8. Rafael M, Zwierewicz M. Do limbo à mesa: o garfo e seu lugar na história da cultura alimentar. III Congresso Internacional de Educação UNIBAVE - Educar na e para a diversidade; 06-08 nov. 2008; Orleans, SC. p. 22-32.

9. Jones T. The history of spoons, forks and Knives. Today I Found [Internet] 3 Out 2013. Disponível em: http://www.todayifoundout.com/index.php/2013/10/history-spoons-forks-knives/

10. Elias N. O processo civilizador: uma história dos costumes. v. 1. Rio de Janeiro: Jorge Zahar; 1994.

11. Fryxell DA. History matters: cutlery. Family tree magazine [Internet] 22 dez. 2010. Disponível em: http://www.familytreemagazine.com/article/mar-2011-history-matters-cutlery

12. Matwick K. Universal cutlery culture: the spoon's diverse history. The Independent Florida Alligator [Internet] Florida, 2 Out. 2014; 109(28):8-9. Disponível em: http://ufdcimages.uflib.ufl.edu/ $\mathrm{UF} / 00 / 02 / 82 / 90 / 01923 / 10-02-2014 . p d f$

13. Lima TA. Pratos e mais pratos: louças domésticas, divisões culturais e limites sociais no Rio de Janeiro, século XIX. Anais do Museu Paulista 1995; 3(1):129-191.

14. Goldsmith S. The rise of the fork [Internet]. New York: Slate; 20 jun. 2012. Disponível em: http:// www.slate.com/articles/arts/design/2012/06/the_history_of_the_fork_when_we_started_using_ forks_and_how_their_design_changed_over_time_.html

15. Snodgrass ME. Encyclopedia of kitchen history. New York: Taylor \& Francis Books, Inc.; 2004.

16. Oliver $\mathrm{S}$. The semiotics of cutlery: eating food symbolically off course [Internet]. Culture Decanted 8 Out. 2014. Disponível em: http://culturedecanted.com/2014/10/08/the-semiotics-of-cutlery-eatingfood-symbolically-off-course/

17. Sideman E. Cutlery [Internet]. How Products Are Made. Disponível em: http://www.madehow. com/Volume-1/Cutlery.html 
18. Nakagawa ADS. A Etiqueta corporativa e o jogo das relações sociais [Internet]. VII Seminário da Associação Nacional Pesquisa e Pós-Graduação em Turismo; 20-21 set. 2010; São Paulo. Disponível em: http://www.anptur.org.br/ocs/index.php/seminario/2010/paper/view/689

19. Ribeiro MTR. O Sentido da sociologia: uma breve abordagem sobre a perspectiva sociológica. Sociedade em Debate 2012; 5(3):49-55.

20. Pellegrino M. História da etiqueta [Internet] Disponível em: http://www.atitudeelegante.com.br/ etiqueta/8-historia-da-etiqueta.html

21. Cobra RQ. Ao restaurante [Internet]. Brasília: Cobra Pages; 2001. Disponível em: http://www.cobra. pages.nom.br/bmp-restaurante.html

22. Soares F, Corção M. Bom gosto à mesa: cultura material e distinção social no Brasil do Século XIX [Internet]. Clio Arqueológica 2012; 27(2). Disponível em: https://www.ufpe.br/clioarq/index. php?option=com_content\&view=article\&id=354\&Itemid $=291$

23. Ceará. Governo do Estado. Escola Estadual de Educação Profissional - EEEP. Ensino médio integrado à educação professional. Curso técnico em nutrição e dietética. Técnica dietética II. Fortaleza: Secretaria de Educação; 2013. 54 p. Disponível em: http://www.seduc.ce.gov.br/images/ APOSTILAS_2012/Nutricao_dietetica/nutricao_e_dietetica_tecnica_dietetica_2.pdf

24. Vanhoenacker M. Put a fork in it: the American way of using fork and knife is inefficient and inelegant. We need a new way [Internet]. Slate. 26 jun. 2013. Disponível em: http://www.slate.com/articles/life/ culturebox/2013/06/fork_and_knife_use_americans_need_to_stop_cutting_and_switching.html

25. Pereira DS. Em busca do refinamento: um estudo antropológico da prática da etiqueta. São Paulo: Annablume; 2006.

26. Cutlery is popular in the West, chopsticks are popular in the east. How did these utensils spread across cultures? [Internet] Reddit: Ask Historians. Disponível em: https://www.reddit.com/r/AskHistorians/ comments/1dghks/cutlery_is_popular_in_the_west_chopsticks_are/

27. Braga IMRMD. Comer e beber no recôncavo baiano: permanências e inovações. Lusíada. História 2014; 2(5/6):223-236.

28. Matarazzo C. Etiqueta sem frescura. São Paulo: Melhoramentos; 1995.

29. Jeudy HP. Philippe Starck: ficção semântica [Internet]. Arcos: Design, cultura material e visualidade 1999; 2:48. Disponível em: http://www.esdi.uerj.br/sobrearcos/p_arcos_2.shtml\#t1

30. Kellner P. Lenguaje de productos. In: Fernández S, Bonsiepe G. Historia del diseño en América Latina y el Caribe. San Pablo: Blücher; 2008.

Recebido: 29/3/2016

Revisado: 05/8/2016

Aceito: 28/8/2016 